\title{
Magnetresonanztomographische Befunde bei Hunden mit Verdacht auf fibrokartilaginären Rückenmarksinfarkt
}

 \\ Aus der ${ }^{1}$ Klinik für Kleintiere (Direktor: Prof. Dr. I. Nolte) und dem ${ }^{3}$ Institut für Pathologie (Direktor: Prof. Dr. W. Baumgärtner, \\ Ph.D.), Stiftung Tierärztliche Hochschule Hannover, sowie dem ${ }^{2}$ Department of Clinical Sciences of Companion Animals \\ (Prof. Dr. J. Rothuizen), Faculty of Veterinary Medicine, Utrecht University, The Netherlands
}

Schlüsselwörter:

fibrokartilaginärer Embolus, Rückenmarksinfarkt, MRT, explosiver Bandscheibenvorfall, Lähmung

\begin{abstract}
Zusammenfassung:
Gegenstand und Ziel: Hunde mit der Verdachtsdiagnose eines fibrokartilaginären Rückenmarksinfarktes wurden retrospektiv im Hinblick auf ihre Befunde der magnetresonanztomographischen Untersuchung (MRT) analysiert. Material und Methoden: Im Zeitraum von Februar 2004 bis Februar 2006 erfüllten 26 Hunde die Einschlusskriterien und gingen in die Studie ein. Bei 22 dieser Hunde erhärtete sich der Verdacht auf einen fibrokartilaginären Rückenmarksinfarkt, von denen sechs Hunde euthanasiert und der Rückenmarksinfarkt histopathologisch bestätigt wurde. Bei den übrigen vier Hunden wurde ein explosiver Bandscheibenvorfall diagnostiziert. Ergebnisse: Von den 26 Hunden zeigten 18 eine intramedulläre Hyperintensität in der T2-gewichteten Spinecho-(SE-)Sequenz, während sich bei acht Hunden das Rückenmark normointens darstellte. Verschiedene Einflussfaktoren auf die Ausbildung einer Hyperintensität wurden evaluiert. Schlussfolgerungen: Der fehlende Nachweis einer intramedullären hyperintensen Läsion in der T2-gewichteten SE-Sequenz schließt einen fibrokartilaginären Rückenmarksinfarkt nicht aus. Die Zeitspanne vom Auftreten der neurologischen Lähmungserscheinungen bis zur Durchführung der MRT-Diagnostik sowie die Größe und Lokalisation der embolisierten Gefäße können einen wichtigen Einfluss auf das Vorhandensein oder Fehlen der Hyperintensität darstellen. Ein explosiver Bandscheibenvorfall kann vergleichbare MRT-Befunde wie der fibrokartilaginäre Rückenmarksinfarkt hervorrufen. Klinische Relevanz: Die präsentierte Studie liefert wichtige Erkenntnisse in der Diagnostik des fibrokartilaginären Rückenmarksinfarktes mittels MRT.
\end{abstract}

Key words:

Fibrocartilaginous embolism, spinal cord infarction, MRI, explosive disc herniation, paralysis

\begin{abstract}
Summary:
Objective: Dogs suspected to suffer from fibrocartilaginous embolization (FCE) were evaluated in a retrospective study in respect to their findings in magnetic resonance imaging (MRI). Material and methods: Of the 26 dogs (February 2004 to February 2006) which met the inclusion criteria for evaluation, suspicion of FCE was substantiated in 22. Six of these dogs were euthanized, and FCE was confirmed histopathologically. The remaining four of the 26 dogs suffered from explosive disc herniation. Results: Eighteen of the 26 dogs showed an intramedullary hyperintensity in T2-weighted spinecho (SE) images, whereas in the remaining eight dogs the spinal cord was normointense. Different factors possibly influencing the presence or absence of hyperintensity were evaluated. Conclusions: The absence of an intramedullary hyperintensity in T2-weighted images does not exclude the existence of FCE. The time lapsed since onset of clinical signs and the MR imaging as well as the size and localization of the embolized blood vessels may have an important impact on the appearance of FCE during MRI. An explosive intervertebral disc herniation can produce the same MRI findings as FCE. Clinical relevance: The study presented provides important insights into the diagnostic approach in dogs with FCE using MRI.
\end{abstract}

Magnetic resonance imaging findings in dogs with suspected fibrocartilaginous embolization

Tierärztl Prax 2007; 35 (K): 163-174

\section{Einleitung}

Der fibrokartilaginäre Embolus, der einen Rückenmarksinfarkt verursacht, im weiteren Text fibrokartilaginärer Rückenmarksinfarkt (fibrocartilaginous embolism $=$ FCE), in der Literatur zum

Eingegangen: 02.06.2006; akzeptiert: 12.11.2006
Teil auch Faserknorpelembolie genannt, stellt beim Hund eine häufige Ursache für akute Lähmungen der Hinter- und Vordergliedmaßen dar (9). Großwüchsige Tiere sind bevorzugt betroffen, doch häufen sich auch Berichte über FCE bei kleineren und auch chondrodystrophen Rassen $(10,12,19,35)$. Sehr häufig besteht eine Asymmetrie der Lähmung, die nach 24 Stunden nicht fortschreitet und bei der nach 24 Stunden keine Schmerzhaftigkeit in der Wirbelsäule festgestellt wird $(11,12,23)$. 
Magnetresonanztomographische Befunde bei Hunden mit Verdacht auf fibrokartilaginären Rückenmarksinfarkt V. M. Stein, F. Wagner, C. Bull, A. Gerdwilker, F. Seehusen, W. Baumgärtner, A. Tipold

Die klinische Diagnose eines FCE ist klassischerweise eine Ausschlussdiagnose (26). Das Zusammenpassen von Anamnese, Signalement, Befunden der neurologischen Untersuchung und fehlende Hinweise auf eine kompressive Läsion in der Röntgendiagnostik, Myelographie und/oder Computertomographie erhärten den Verdacht auf das Vorliegen eines FCE. Die definitive Diagnose ist jedoch der histopathologischen Untersuchung vorbehalten.

Die Magnetresonanztomographie (MRT) wird in der Humanmedizin seit 1989 bei Verdacht auf einen Rückenmarksinfarkt zur Diagnostik eingesetzt (7). Obwohl verschiedene Autoren die MRT auch zur Diagnostik des Rückenmarksinfarktes beim Hund empfehlen $(4,9,26)$, gibt es bisher kaum Beschreibungen der Befunde in der Veterinärmedizin. Die Magnetresonanztomographie könnte ein neues wertvolles Werkzeug für die klinische Diagnostik des FCE darstellen.

Diese Studie beschreibt die Befunde der MRT-Diagnostik von Hunden mit der Verdachtsdiagnose eines fibrokartilaginären
Rückenmarksinfarktes. Die Diagnose wurde bei sechs Hunden histopathologisch bestätigt.

\section{Material und Methoden}

\section{Patienten}

Die Daten der Hunde, die zwischen Februar 2004 und Februar 2006 Patienten der Klinik für Kleintiere der Stiftung Tierärztliche Hochschule Hannover waren, wurden retrospektiv evaluiert. Aufnahme in die Studie fanden Hunde, die akute oder perakute Lähmungen der Hinter- und/oder Vordergliedmaßen, häufig mit einer Asymmetrie der Lähmungen aufwiesen und bei denen mittels MRT kompressive Rückenmarkserkrankungen, wie Bandscheibenvorfälle oder Wirbelluxationen und -subluxationen, ausgeschlossen wurden. Hunde, die vor der Überweisung mehr als einmal Glukokortikosteroide erhalten hatten, gingen nicht in die Studie ein, da eine Beeinflussung der magnetresonanztomographischen Befunde zu vermuten war. Hunde mit Blutungen im Rückenmark wurden aufgrund der unterschiedlichen Darstellung in der MRT mit einer Hyperinten-

Tab. 1 Zusammenfassung der Daten der Hunde mit der Verdachtsdiagnose Rückenmarksinfarkt ( $\mathrm{n}=22)$. $\mathrm{J}=\mathrm{Jahre}, \mathrm{M}=\mathrm{Monate}, \mathrm{m}=$ männlich, $\mathrm{w}=$ weiblich, $\mathrm{k}=$ kastriert, UMN = unteres Motoneuronsystem, OMN = oberes Motoneuronsystem, vo. = vorne, hi. = hinten, re. = rechts, li. = links. In Klammern sind die betroffenen Rückenmarkssegmente definiert. Die schattierten Zeilen kennzeichnen die Hunde, bei denen eine histopathologische Untersuchung erfolgte. Eine Seitenbetonung wurde bei plegischen Tieren aufgrund der Reflexantwort festgestellt.

\begin{tabular}{|c|c|c|c|c|c|}
\hline Fall-Nr. & Rasse & Alter & $\begin{array}{l}\text { Gewicht } \\
\text { (kg) }\end{array}$ & Geschlecht & Gangstörung und neuroanatomische Lokalisation \\
\hline 1 & Berner Sennenhund & $5 \mathrm{~J}$ & 46 & $\mathrm{~m}$ & Paraplegie mit li. Seitenbetonung, UMN hi. (L4-S3) \\
\hline 2 & Briard & $6 \mathrm{~J}, 11 \mathrm{M}$ & 37,5 & w & Paraplegie, UMN hi. (L4-S3) \\
\hline 3 & Mastino Neapolitano & $6 \mathrm{~J}, 6 \mathrm{M}$ & 58 & wk & Paraparese mit re. Seitenbetonung, OMN hi. (T3-L3) \\
\hline 4 & Deutsch Drahthaar & $7 \mathrm{~J}, 8 \mathrm{M}$ & 42 & $\mathrm{~m}$ & Paraplegie mit li. Seitenbetonung, UMN hi. (L4-S3) \\
\hline 5 & Mischling & $8 \mathrm{~J}, 10 \mathrm{M}$ & 23 & w & hgr. Paraparese, OMN hi. (T3-L3) \\
\hline 6 & Mischling & $8 \mathrm{~J}$ & 20 & $\mathrm{~m}$ & Paraplegie, OMN hi. (T3-L3) \\
\hline 7 & Yorkshire Terrier & $3 \mathrm{~J}, 10 \mathrm{M}$ & 3,5 & $\mathrm{~m}$ & hgr. Tetraparese mit re. Seitenbetonung, UMN vo. (C6-T2) \\
\hline 8 & Berner Sennenhund & $9 \mathrm{~J}, 8 \mathrm{M}$ & 42 & $\mathrm{~m}$ & hgr. Tetraparese, UMN vo. (C6-T2) \\
\hline 9 & Mischling & $6 \mathrm{~J}$ & 32 & $\mathrm{~m}$ & Paraparese mit li. Seitenbetonung, OMN hi. (T3-L3) \\
\hline 10 & Berner Sennenhund & $1 \mathrm{~J}, 6 \mathrm{M}$ & 30 & w & Monoparese li. Hintergliedmaße, UMN hi. (L4-S3) \\
\hline 11 & Labrador Retriever & $7 \mathrm{~J}, 10 \mathrm{M}$ & 34 & $\mathrm{mk}$ & Paraparese mit re. Seitenbetonung (Plegie), OMN hi. (T3-L3) \\
\hline 12 & Labrador Retriever & $3 \mathrm{~J}, 11 \mathrm{M}$ & 45 & $\mathrm{~m}$ & Paraparese mit re. Seitenbetonung (Plegie), OMN hi. (T3-L3) \\
\hline 13 & Hovawart & $8 \mathrm{~J}$ & 36,5 & w & Paraparese mit re. Seitenbetonung, UMN hi. (L4-S3) \\
\hline 14 & Golden Retriever & $6 \mathrm{~J}, 7 \mathrm{M}$ & 40 & $\mathrm{mk}$ & Paraparese mit li. Seitenbetonung, UMN hi. (L4-S3) \\
\hline 15 & Whippet & $6 \mathrm{~J}, 11 \mathrm{M}$ & 14,5 & $\mathrm{~m}$ & Tetraparese mit li. Seitenbetonung, 0MN vo. (C1-C5) \\
\hline 16 & Riesenschnauzer & $5 \mathrm{~J}, 7 \mathrm{M}$ & 36 & w & Tetraplegie mit re. Seitenbetonung, UMN vo. (C6-T2) \\
\hline 17 & Labrador-Mischling & $8 \mathrm{~J}, 3 \mathrm{M}$ & 26 & $\mathrm{~m}$ & Paraplegie mit re. Seitenbetonung, OMN hi. (T3-L3) \\
\hline 18 & Golden Retriever & $4 \mathrm{~J}, 10 \mathrm{M}$ & 29 & w & Paraplegie, OMN hi. (T3-L3) \\
\hline 19 & Kanadischer Schäferhund & $5 \mathrm{~J}, 11 \mathrm{M}$ & 36 & w & Paraplegie, UMN hi. (L4-S3) \\
\hline 20 & Border Collie & $6 \mathrm{~J}, 8 \mathrm{M}$ & 20,5 & $\mathrm{~m}$ & Paraparese mit re. Seitenbetonung, UMN hi. (L4-S3) \\
\hline 21 & Jindo & $12 \mathrm{~J}, 1 \mathrm{M}$ & 20 & w & Paraparese mit li. Seitenbetonung, OMN hi. (T3-L3) \\
\hline 22 & Landseer & $5 \mathrm{~J}, 10 \mathrm{M}$ & 60 & $\mathrm{~m}$ & hgr. Paraparese, UMN hi. (L4-S3) \\
\hline
\end{tabular}


Magnetresonanztomographische Befunde bei Hunden mit Verdacht auf fibrokartilaginären Rückenmarksinfarkt V. M. Stein, F. Wagner, C. Bull, A. Gerdwilker, F. Seehusen, W. Baumgärtner, A. Tipold

sität in der T1-gewichteten Sequenz (33) identifiziert. Intramedulläre Tumoren konnten durch ihr Wachstum mit Gewebsverdrängung oder einen Masseneffekt sowie durch Anreicherung von Kontrastmittel (22) differenziert werden.

Im zweijährigen Beobachtungszeitraum ließen sich 49 Hunde identifizieren, die perakute bis akute Lähmungen, oftmals mit einer Seitenbetonung gezeigt hatten und magnetresonanztomographisch untersucht worden waren. In 23 Fällen führte der Nachweis kompressiver Läsionen in der MRT zum Studienausschluss. Von den verbliebenen 26 Hunden bestand bei 22 der Verdacht auf einen fibrokartilaginären Rückenmarksinfarkt. Sechs dieser Hunde wurden euthanasiert und histopathologisch untersucht. Bei den vier übrigen Hunden ergaben intraoperative sowie histopathologische Befunde die Diagnose eines explosiven Bandscheibenvorfalls. Die MRT-Befunde dieser vier Tiere wurden mit denen der 22 Hunde mit fibrokartilaginärem Rückenmarksinfarkt verglichen.

\section{Neurologische Untersuchung}

Bei allen Patienten erfolgte eine neurologische Untersuchung zur Lokalisierung der Läsion. Alle Hunde zeigten perakute bis akute neurologische Ausfallerscheinungen, die im zervikalen, thorakalen oder lumbalen Rückenmark lokalisiert wurden (Tab. 1). Während des stationären Aufenthalts der Tiere fand zur Evaluierung des klinischen Verlaufs täglich eine neurologische Kontrolluntersuchung statt.

\section{Magnetresonanztomographie}

Die Magnetresonanztomographie (Magnetom Impact Plus, 1.0 Tesla, Siemens, Erlangen) wurde in Allgemeinanästhesie durchgeführt. Zur Narkoseeinleitung erhielten die Hunde eine intravenöse Injektion von Propofol $\left(\mathrm{Narcofol}^{\circledR}\right.$, cppharma, Burgdorf, Dosierung nach Wirkung) und Diazepam (diazepam-ratiopharm $^{\circledR}$, Fa. Ratiopharm GmbH, Ulm, 1 mg/kg KM i. v., maximal $30 \mathrm{mg}$ ). Die Erhaltung der Allgemeinanästhesie wurde nach Intubation mit Isofluran (Isofluran-Baxter ${ }^{\circledR}$, Baxter Deutschland $\mathrm{GmbH}$, Unterschleißheim) in einem Gemisch

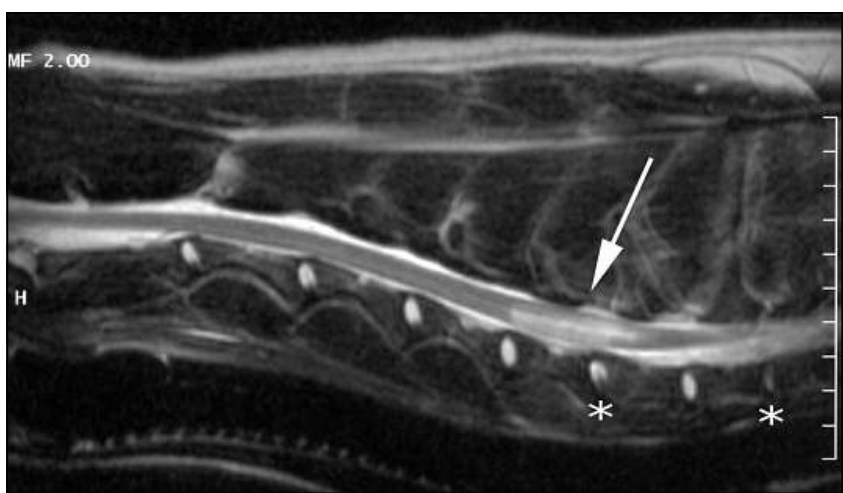

Abb. 1 Magnetresonanztomographische Darstellung der zervikalen Wirbelsäule in der sagittalen Projektion der T2-gewichteten Spinecho-(SE-)Sequenz von Hund Nr. 16. Von C6 bis C7 ist die intramedulläre Hyperintensität deutlich sichtbar (Pfeil). Die Nuclei pulposi von C6/7 (Stern) und Th1/2 (Stern) erscheinen hypointens und der Discus intervertebralis von $\mathrm{C6} / 7$ erscheint kleiner als die benachbarten Disken.

aus Sauerstoff und atmosphärischer Luft fortgeführt (Narkosegerät mit assistierter Beatmung, Titus, Fa. Dräger Medizintechnik GmbH, Lübeck). Bei allen Patienten wurden T2-gewichtete Turbo-Spinecho-(TSE-)Sequenzen in sagittalen $(\mathrm{TR}=$ Repetitionszeit in Millisekunden: $4700 \mathrm{~ms}, \mathrm{TE}=$ Echozeit in Millisekunden: $112 \mathrm{~ms}$, Flipwinkel $180^{\circ}$ ) und transversalen (TR 3458, TE $96 \mathrm{~ms}$, Flipwinkel $180^{\circ}$ ) Projektionsebenen erfasst, um die in der Literatur beschriebene intramedulläre Hyperintensität darzustellen. In einzelnen Fällen mit intramedullärer Hyperintensität in der T2-Gewichtung wurden zusätzlich T1-Spinecho (SE)

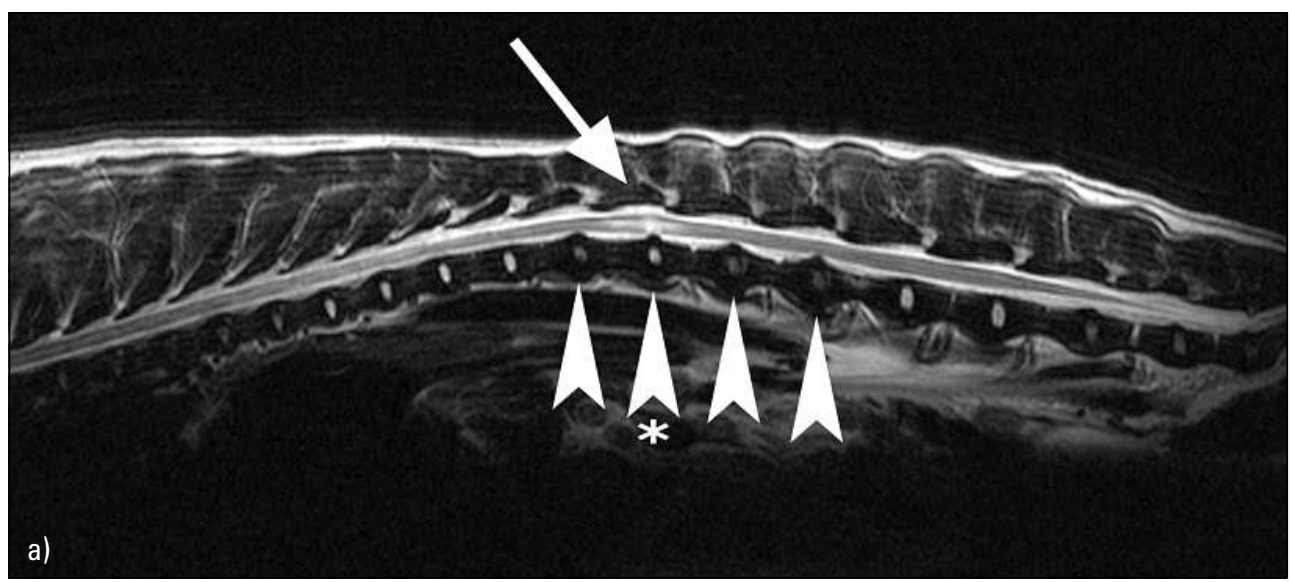

Abb. 2 Magnetresonanztomographische Darstellung der thorakolumbalen Wirbelsäule in der sagittalen (a) und transversalen (b) Projektionsebene der T2-gewichteten TSE-Sequenz von Hund Nr. 17. a) In der sagittalen Projektionsebene ist oberhalb des Intervertebralspaltes von Th13 und L1 die intramedulläre hyperintense Läsion deutlich sichtbar (Pfeil). Die Nuclei pulposi von Th12/13, L1/2 und L2/3 sind hypointens (Pfeilspitzen), der Diskus von Th13/L1 erscheint kleiner als die benachbarten Disken (Pfeilspitze mit Stern). b) In der transversalen Projektion stellt sich vor allem die graue Substanz hyperintens dar.

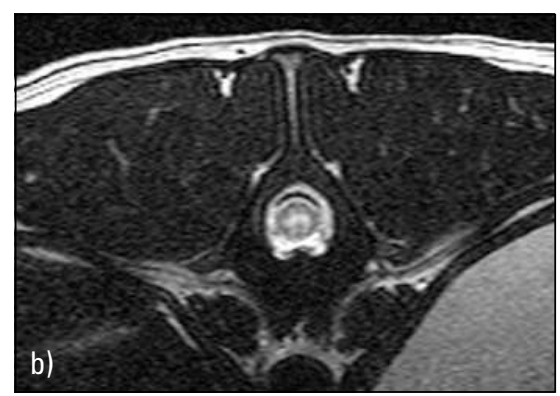


Magnetresonanztomographische Befunde bei Hunden mit Verdacht auf fibrokartilaginären Rückenmarksinfarkt V. M. Stein, F. Wagner, C. Bull, A. Gerdwilker, F. Seehusen, W. Baumgärtner, A. Tipold

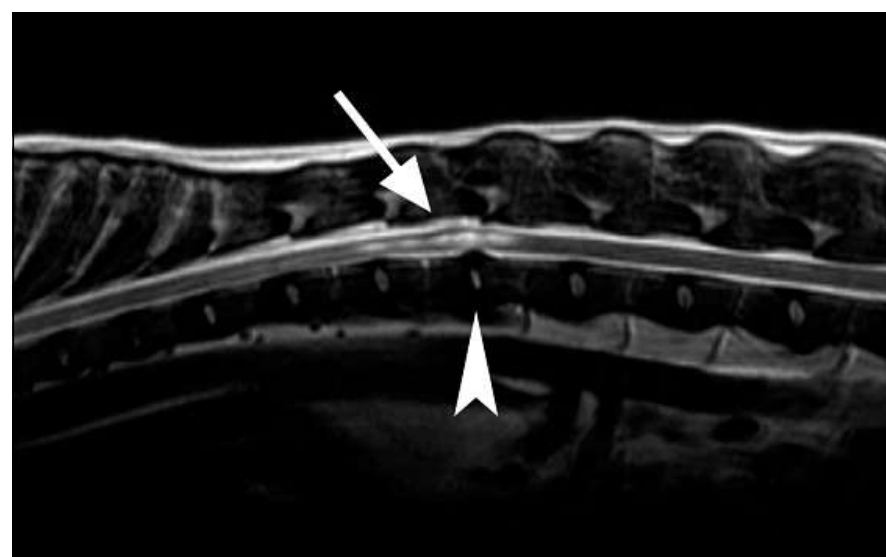

a)

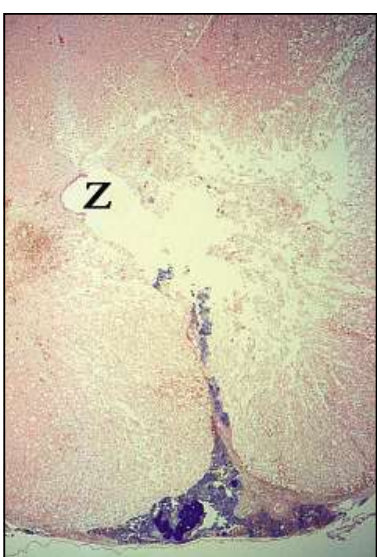

b)

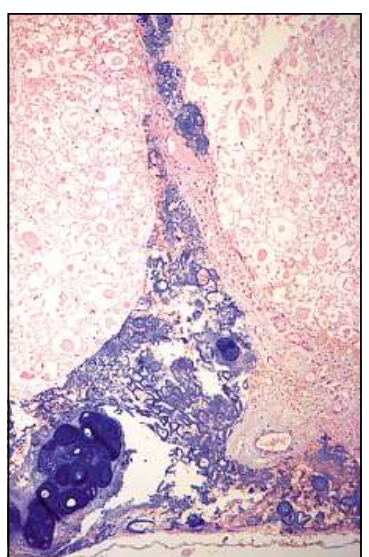

c)

Abb. 3 a) Magnetresonanztomographische Darstellung der thorakolumbalen Wirbelsäule in der sagittalen Projektionsebene in der T2-gewichteten TSE-Sequenz eines Hundes mit einem explosiven Bandscheibenvorfall. Bei Th13/L1 ist deutlich die intramedulläre Hyperintensität sichtbar (Pfeil), der Nucleus pulposus von Th13/L1 erscheint mäßig hypointens und kleiner als die benachbarten Disken (Pfeilspitze). b) Histopathologische Darstellung in der Alzianblau-Färbung ( $25 \times$, Originalvergrößerung). Hochgradige Malazie in den ventromedialen Funiculi der weißen Substanz mit Nachweis von dunkelblau gefärbtem, explosiv vorgefallenem Diskusmaterial. c) Ausschnitt aus b) $(100 \times$, Originalvergrößerung). $Z$ = Zentralkanal.

(TR $330 \mathrm{~ms}$, TE $12 \mathrm{~ms}$, Flipwinkel 90) und T1-SE-gewichtete Sequenzen nach Gabe von Gadolinium-Dimeglumine-Kontrastmittel (GdDTPA, Magnevist ${ }^{\circledR}$, Schering Deutschland $\mathrm{GmbH}$, Berlin; $0,2 \mathrm{mmol} / \mathrm{kg} \mathrm{KM} \mathrm{i.} \mathrm{v.)} \mathrm{angefertigt,} \mathrm{um} \mathrm{die}$ Läsion näher zu charakterisieren. Die Schichtdicke betrug für die Transversalschnitte $4 \mathrm{~mm}$ und für die Sagittalschnitte $3 \mathrm{~mm}$.

Es wurde versucht, die MRT-Befunde mit der Gefäßtopographie respektive der Lokalisation im Rückenmark, der einmaligen Vorbehandlung mit Glukokortikosteroiden, der Zeitspanne zwischen dem Beginn der neurologischen Symptome und der Durchführung der MRT, der Schwere der neurologischen Lähmungserscheinungen und dem klinischen Verlauf zu korrelieren.

\section{Ergebnisse}

Bei 22 der 26 in die Studie aufgenommenen Hunde wurde die Verdachtsdiagnose eines fibrokartilaginären Rückenmarksinfarktes gestellt. Bei den übrigen vier Hunden ergab sich die Diagnose explosiver Bandscheibenvorfälle. Die Untersuchungsergebnisse der 22 Hunde mit Verdacht auf einen fibrokartilaginären Rückenmarksinfarkt werden im Folgenden präsentiert. Des Weiteren erfolgt ein Vergleich der MRT-Befunde zwischen den beiden Gruppen (Rückenmarksinfarkt, Bandscheibenvorfall).

Das mittlere Alter der Hunde zum Zeitpunkt der Präsentation betrug 6,5 Jahre (arithmetischer Mittelwert; Bereich: 1,5 bis 12 Jahre). Das Geschlechterverhältnis stellte sich wie folgt dar: 13 Hunde waren männlich (davon zwei kastriert) und neun Hunde weiblich (davon einer kastriert). Zwanzig Patienten zählten zu den großen Hunden ( $\geq 20 \mathrm{~kg} \mathrm{KM}$ ), während nur zwei Hunde ein Körpergewicht von unter $20 \mathrm{~kg}$ aufwiesen. Häufigste Rassen waren Berner Sennenhund (3), Labrador Retriever (2) und Golden Retriever (2). In jeweils einem Fall handelte es sich um folgende Rassen: Briard, Mastino Neapolitano, Deutsch Drahthaar, Yorkshire Terrier, Hovawart, Whippet, Riesenschnauzer, Amerikanisch-Ka- nadischer Schäferhund, Border Collie, Jindo und Landseer. Des Weiteren fanden sich unter den Patienten vier Mischlingshunde.

Die häufigsten neuroanatomischen Lokalisationen der Läsionen stellten das obere motorische Neuronsystem hinten $(\mathrm{OMN}, \mathrm{T} 3-\mathrm{L} 3, \mathrm{n}=9)$ und das untere motorische Neuronsystem hinten (UMN, L4-S3, n = 9) dar. Somit ergab sich als häufigste beobachtete neurologische Lähmungserscheinung eine Paraparese oder -plegie. Das zervikale Rückenmark war nur in vier Fällen betroffen, die sich klinisch als Tetraparese und in einem Fall (Nr. 16) als Tetraplegie manifestierten. Dabei wurde die Läsion lediglich einmal auf das obere motorische Neuronsystem (OMN, C1-5) und dreimal auf das untere motorische Neuronsystem (UMN, C6-T2) lokalisiert. In etwa 70\% der Fälle konnte eine Asymmetrie der neurologischen Lähmungserscheinungen beobachtet werden (Tab. 1).

Die MRT-Untersuchung ergab in der T2-gewichteten TSESequenz bei 14 Hunden eine fokale intramedulläre hyperintense Läsion (Abb. 1-3, Tab. 2), während sich bei acht Hunden das Rückenmark im gesamten Bereich der neuroanatomischen Lokalisation der Läsion normointens darstellte (Tab. 2). In den Transversalschnitten zeigte sich eine gute Korrelation zwischen asymmetrischer Hyperintensität und den asymmetrischen neurologischen Defiziten (vgl. Tab. 1, 2). Bei acht der 14 Hunde mit intramedullärer Hyperintensität bestand eine klinische Seitenbetonung, die sich bei sieben Tieren auch magnetresonanztomographisch nachvollziehen ließ. Die anderen sechs Hunde wiesen klinisch keine Seitenbetonung der neurologischen Defizite auf. Bei fünf dieser sechs Hunde betraf die intramedulläre Hyperintensität relativ gleichmäßig beide Seiten des Rückenmarks (vgl. Tab. 1,2).

In sieben Fällen mit einer intramedullären Hyperintensität in der T2-gewichteten TSE-Sequenz wurde eine T1-gewichtete SESequenz des Rückenmarks durchgeführt. In dieser Darstellung er- 
Tab. 2 MRT-Befunde in der T2-gewichteten TSE-Sequenz in der sagittalen und transversalen Projektionsebene unter Berücksichtigung der Zeitspanne von der Initiation der neurologischen Symptome bis zur Durchführung der MRT-Diagnostik. - = Untersuchung nicht durchgeführt. Die schattierten Zeilen kennzeichnen die Hunde, bei denen keine Hyperintensität in der T2-gewichteten TSE-Sequenz gefunden wurde.

\begin{tabular}{|c|c|c|c|}
\hline \multirow[t]{2}{*}{ Fall-Nr. } & \multirow{2}{*}{$\begin{array}{l}\text { Dauer } \\
\text { bis MRT }\end{array}$} & \multicolumn{2}{|l|}{ MRT-Befunde in der T2-gewichteten Spinecho-(SE-)Sequenz } \\
\hline & & sagittal & transversal \\
\hline 12 & $<8 \mathrm{~h}$ & $\begin{array}{l}\text { intramedulläre Hyperintensität bei L2/3, Discus interver- } \\
\text { tebralis von L2/3 hypointens }\end{array}$ & $\begin{array}{l}\text { intramedulläre Hyperintensität vorwiegend der } \\
\text { grauen Substanz bei L2/3 mit rechter Seitenbetonung }\end{array}$ \\
\hline 18 & $<8 \mathrm{~h}$ & intramedulläre Hyperintensität von Th9 bis Th11 & $\begin{array}{l}\text { diffuse intramedulläre Hyperintensität von Th9-11 } \\
\text { ohne Seitenbetonung }\end{array}$ \\
\hline 2 & $<24 \mathrm{~h}$ & intramedulläre Hyperintensität bei $L 4 / 5$ mit Rechtsbetonung & $\begin{array}{l}\text { intramedulläre Hyperintensität der grauen und wei- } \\
\text { ßen Substanz der rechten Seite }\end{array}$ \\
\hline 3 & $<24 \mathrm{~h}$ & $\begin{array}{l}\text { normointenses Rückenmark, multiple hypointense degene- } \\
\text { rierte Disci intervertebrales im Bereich der BWS }\end{array}$ & - \\
\hline 4 & $<24 \mathrm{~h}$ & $\begin{array}{l}\text { normointenses Rückenmark, Disci intervertebrales von } \\
\text { L6/7 und L7/S1 ggr. hypointens }\end{array}$ & - \\
\hline 5 & $<24 \mathrm{~h}$ & $\begin{array}{l}\text { intramedulläre Hyperintensität bei L2, Discus interverte- } \\
\text { bralis von L2/3 ggr. hypointens }\end{array}$ & $\begin{array}{l}\text { Hyperintensität der grauen Substanz bei L2/3 ohne } \\
\text { Seitenbetonung }\end{array}$ \\
\hline 6 & $<24 \mathrm{~h}$ & $\begin{array}{l}\text { intramedulläre Hyperintensität bei Th12/13, Discus inter- } \\
\text { vertebralis von Th12/13 ggr. kleiner erscheinend als die } \\
\text { benachbarten, Disken kranial von Th10 hypointens }\end{array}$ & $\begin{array}{l}\text { Hyperintensität der grauen Substanz bei Th12/13 } \\
\text { ohne Seitenbetonung }\end{array}$ \\
\hline 14 & $<24 \mathrm{~h}$ & $\begin{array}{l}\text { intramedulläre Hyperintensität bei } L 3 / 4 \text {, Discus interver- } \\
\text { tebralis von } L 1 / 2 \text { hypointens }\end{array}$ & $\begin{array}{l}\text { diffuse intramedulläre Hyperintensität der grauen } \\
\text { und weißen Substanz mit linker Seitenbetonung }\end{array}$ \\
\hline 19 & $<24 \mathrm{~h}$ & $\begin{array}{l}\text { intramedulläre Hyperintensität von L1 bis L4, Disci inter- } \\
\text { vertebrales von L6/7 und L7/S1 hypointens, von L3/4 mäßig } \\
\text { hypointens }\end{array}$ & $\begin{array}{l}\text { intramedulläre Hyperintensität vorwiegend der } \\
\text { grauen Substanz ohne Seitenbetonung }\end{array}$ \\
\hline 20 & $<24 \mathrm{~h}$ & $\begin{array}{l}\text { normointenses Rückenmark, multiple hypointense Disci } \\
\text { intervertebrales kranial von Th10 und kaudal von Th13 }\end{array}$ & - \\
\hline 22 & $<24 \mathrm{~h}$ & intramedulläre Hyperintensität von L4 bis L6 & $\begin{array}{l}\text { Hyperintensität vor allem der grauen Substanz ohne } \\
\text { Seitenbetonung }\end{array}$ \\
\hline 9 & $24-48 \mathrm{~h}$ & $\begin{array}{l}\text { normointenses Rückenmark, Discus intervertebralis bei } \\
\text { Th12/13 hypointens }\end{array}$ & - \\
\hline 10 & $24-48 \mathrm{~h}$ & normointenses Rückenmark & normointenses Rückenmark \\
\hline 11 & $24-48 \mathrm{~h}$ & $\begin{array}{l}\text { intramedulläre Hyperintensität bei Th13/L1, Discus inter- } \\
\text { vertebralis bei Th13/L1 ggr. kleiner erscheinend, Disken } \\
\text { von } \mathrm{L} 3 / 4 \text { und } \mathrm{L} 4 / 5 \text { hypointens }\end{array}$ & $\begin{array}{l}\text { intramedulläre wolkige Hyperintensität bei Th13/L1 } \\
\text { rechtsseitig im ventralen Quadranten }\end{array}$ \\
\hline 16 & $24-48 \mathrm{~h}$ & $\begin{array}{l}\text { intramedulläre Hyperintensität bei C6 bis C7, Disci inter- } \\
\text { vertebrales von C6/7 und Th1/2 hypointens, C6/7 kleiner } \\
\text { erscheinend }\end{array}$ & $\begin{array}{l}\text { intramedulläre Hyperintensität mit rechter Seiten- } \\
\text { betonung }\end{array}$ \\
\hline 17 & $24-48 \mathrm{~h}$ & $\begin{array}{l}\text { intramedulläre Hyperintensität bei Th13/L1, Disci interver- } \\
\text { tebrales von Th12/13, L1/2 und L2/3 hypointens }\end{array}$ & $\begin{array}{l}\text { intramedulläre Hyperintensität vorwiegend der } \\
\text { grauen Substanz bei Th13/L1 ohne Seitenbetonung }\end{array}$ \\
\hline 1 & $>48 \mathrm{~h}$ & $\begin{array}{l}\text { intramedulläre Hyperintensität von L2-L5 mit Linksbeto- } \\
\text { nung, kein Hinweis auf Nucleusdegeneration }\end{array}$ & $\begin{array}{l}\text { intramedulläre Hyperintensität linksseitig dorso- } \\
\text { lateral }\end{array}$ \\
\hline 7 & $>48 \mathrm{~h}$ & intramedulläre Hyperintensität bei C5/6 & $\begin{array}{l}\text { intramedulläre Hyperintensität der grauen und wei- } \\
\text { ßen Substanz bei C5/6 mit rechter Seitenbetonung }\end{array}$ \\
\hline 8 & $>48 \mathrm{~h}$ & $\begin{array}{l}\text { normointenses Rückenmark, Disci intervertebrales der } \\
\text { HWS generalisiert relativ hypointens }\end{array}$ & normointenses Rückenmark \\
\hline 13 & $>48 \mathrm{~h}$ & $\begin{array}{l}\text { normointenses Rückenmark, Disci intervertebrales von } \\
L 1 / 2 \text {, } L 2 / 3 \text { und } L 3 / 4 \text {, sowie von } L 7 / S 1 \text { mäßig hypointens }\end{array}$ & normointenses Rückenmark \\
\hline 15 & $>48 \mathrm{~h}$ & $\begin{array}{l}\text { normointenses Rückenmark, Disci intervertebrales von } \\
\text { C5/6 und } C 6 / 7 \text { hypointens }\end{array}$ & normointenses Rückenmark \\
\hline 21 & $>48 \mathrm{~h}$ & $\begin{array}{l}\text { intramedulläre Hyperintensität bei Th11-Th12 mit Links- } \\
\text { betonung, multiple Disci intervertebrales kranial von Th11 } \\
\text { hypointens }\end{array}$ & $\begin{array}{l}\text { diffuse intramedulläre Hyperintensität der grauen } \\
\text { und weißen Substanz der linken Seite }\end{array}$ \\
\hline
\end{tabular}


Magnetresonanztomographische Befunde bei Hunden mit Verdacht auf fibrokartilaginären Rückenmarksinfarkt V. M. Stein, F. Wagner, C. Bull, A. Gerdwilker, F. Seehusen, W. Baumgärtner, A. Tipold

schien das Rückenmark in fünf Fällen normointens (Hund Nr. 6, $7,14,17,22)$, d. h. es war keine Läsion im Bereich der klinisch definierten neuroanatomischen Lokalisation respektive der Hyperintensität in der T2-gewichteten TSE-Sequenz sichtbar. Dagegen ergab sich an dieser Stelle bei zwei Patienten eine hypointense Läsion (Hund Nr. 11 und 21). Für die unterschiedliche Darstellung der Läsion in der T1 ließ sich keine zeitliche Abhängigkeit feststellen. Nach intravenöser Gabe von GdDTPA zeigte sich im Allgemeinen keine oder lediglich eine sehr geringgradige Kontrastmittelanreicherung im Bereich der intramedullären Hyperintensität.

Um Aufschluss über die unterschiedliche MRT-Darstellung der Läsionen zu erhalten, wurden verschiedene Einflussfaktoren evaluiert und versucht, diese mit dem Vorhandensein oder Fehlen einer intramedullären Hyperintensität in der T2-gewichteten TSE-Sequenz zu korrelieren.

Von den 14 Patienten mit einer Hyperintensität in der T2-gewichteten TSE-Sequenz zeigten sieben Hunde einen vollständigen Verlust der Motorik (Plegie) und sieben Hunde einen partiellen Motorikausfall (Parese). Bei den acht Patienten mit T2-normointensem Rückenmark wies nur ein Hund eine Plegie und sieben Tiere eine Parese auf (vgl. Tab. 1, 2). Aufgrund der unterschiedlichen Gefäßverteilung entlang des Rückenmarks könnten Hunde mit einer neuroanatomischen Lokalisation ihrer Läsion im oberen motorischen Neuronsystem (OMN) vorne (C1-C5) und hinten (T3-L3) häufiger ein Ödem entwickeln als Patienten mit einer Läsion im unteren motorischen Neuronsystem (UMN, C6-T2 oder L4-S3). Von den 10 Hunden mit einer Läsion im OMN wiesen sieben Tiere ein intramedulläres Ödem und lediglich drei Hunde ein normointenses Rückenmark auf. Zwölf Hunde zeigten eine Läsion im UMN, wovon bei sieben eine hyperintense Läsion und bei fünf Hunden ein normointenses Rückenmark darstellbar war (vgl. Tab. 1, 2).

Fünfzehn Hunde waren vor der Überweisung an die Klinik einmalig mit Glukokortikosteroiden vorbehandelt worden. Bei neun dieser Hunde bestand in der T2-gewichteten TSE-Sequenz eine charakteristische hyperintense Läsion des Rückenmarks, während bei sechs Tieren keine Hyperintensität vorlag. Somit konnte zwischen einer Vorbehandlung mit Steroiden und dem Vorhandensein respektive der Abwesenheit einer hyperintensen Läsion in derT2-gewichteten TSE-Sequenz keine Korrelation festgestellt werden.

Die Zeitspanne zwischen dem Auftreten neurologischer Symptome und der MRT-Diagnostik schien keinen Einfluss darauf zu haben, ob sich in der durch die neurologische Untersuchung bestimmten Lokalisation im Rückenmark in der T2-gewichteten TSE-Sequenz eine hyperintense Läsion befand oder sich das Gewebe normointens darstellte. Elf der 22 Hunde wurden am Tag der Initiation der neurologischen Symptome überwiesen und die MRT fand somit innerhalb von 24 Stunden statt. Bei fünf weiteren Hunden erfolgte sie innerhalb von 48 Stunden und bei den verbliebenen sechs Tieren nach Ablauf von 48 Stunden. Acht der 11 binnen 24 Stunden untersuchten Hunde wiesen in der T2 eine intramedulläre Hyperintensität auf, die anderen drei ein nor- mointenses Rückenmark. Bei der MRT-Diagnostik innerhalb von 24-48 Stunden wurden drei Hunde mit und zwei Hunde ohne Hyperintensität erfasst. Von den mehr als 48 Stunden nach Beginn der Symptomatik untersuchten Patienten wiesen drei Hunde eine hyperintense Läsion auf und drei Hunde keine. Somit ließ sich zwischen der Zeitspanne vom Beginn der neurologischen Symptome bis zur MRT-Diagnostik und den Befunden der MRT keine Korrelation nachweisen.

Ein neurologischer Verlauf konnte bei 13 Patienten evaluiert werden, während bei neun Patienten keine Kontrolluntersuchung möglich war. Bei Letzteren handelte es sich um ein in der Allgemeinanästhesie verstorbenes Tier, sechs auf Wunsch des Besitzers euthanasierte Hunde sowie zwei Hunde, die nicht nachuntersucht werden konnten. Acht der 13 nachuntersuchten Patienten wiesen eine intramedulläre hyperintense Läsion in der T2 auf und zeigten im Mittel nach durchschnittlich 12,5 Tagen eine neurologische Besserung (d. h. einsetzende Spontanbewegung bei zuvor plegischen Patienten oder selbständiges Laufen bei einer Parese, das zuvor nur mit Unterstützung möglich war). Der Heilungsverlauf für fünf Hunde mit normointensem Rückenmark in der T2 schien tendenziell kürzer zu sein und betrug im Durchschnitt 4,2 Tage.

In der T2-gewichteten TSE-Sequenz zeigten sich bei 17 Hunden als Hinweis für eine Degeneration eine verringerte Signalintensität der Nuclei pulposi und zum Teil ein geringeres Volumen im Vergleich zu benachbarten Nuclei (Tab. 2). Bei sieben Hunden war der Nucleus direkt unterhalb der hyperintensen Läsion betroffen, bei sechs Tieren konnte dies nicht bestimmt werden, da sich das Rückenmark normointens darstellte. Die degenerierten Nuclei pulposi befanden sich bei diesen sechs Tieren jedoch im Bereich der klinisch definierten neuroanatomischen Lokalisation der Läsion. Bei vier Patienten waren die degenerierten Nuclei mehr als zwei Zwischenwirbelspalten von der Rückenmarksläsion entfernt. Keine Anzeichen für eine Nucleusdegeneration ergaben sich bei fünf Hunden (Tab. 2).

Alle vier Hunde, bei denen anhand intraoperativer $(n=2)$ oder histopathologischer Befunde $(\mathrm{n}=2)$ ein explosiver Diskusprolaps als Lähmungsursache nachgewiesen wurde, zeigten - wie die Mehrzahl der Tiere mit Verdacht auf fibrokartilaginären Rückenmarksinfarkt - in der T2-gewichteten TSE-Sequenz eine intramedulläre Hyperintensität. Diese befand sich bei zwei Tieren oberhalb des Discus intervertebralis von Th12/13 und bei den anderen beiden oberhalb des Diskus von Th13/L1 (Abb. 3). In zwei der vier Fälle erschienen die benannten Nuclei pulposi hypointens und kleiner als die benachbarten. Eine T1-gewichtete SE-Sequenz wurde bei zwei Hunden durchgeführt. In dieser Darstellung zeigte sich die medulläre Läsion in einem Fall hypo-, in dem anderen Fall isointens zum umgebenden Rückenmarksgewebe.

Eine histopathologische Untersuchung erfolgte bei sechs der 22 Hunde mit Verdacht auf einen fibrokartilaginären Infarkt und bei zwei der Hunde mit explosivem Bandscheibenvorfall. In der erstgenannten Gruppe konnte bei drei Hunden eine akute ischämische Myelomalazie festgestellt und das fibrokartilaginäre Material in arteriellen Gefäßen nachgewiesen werden (Hund 

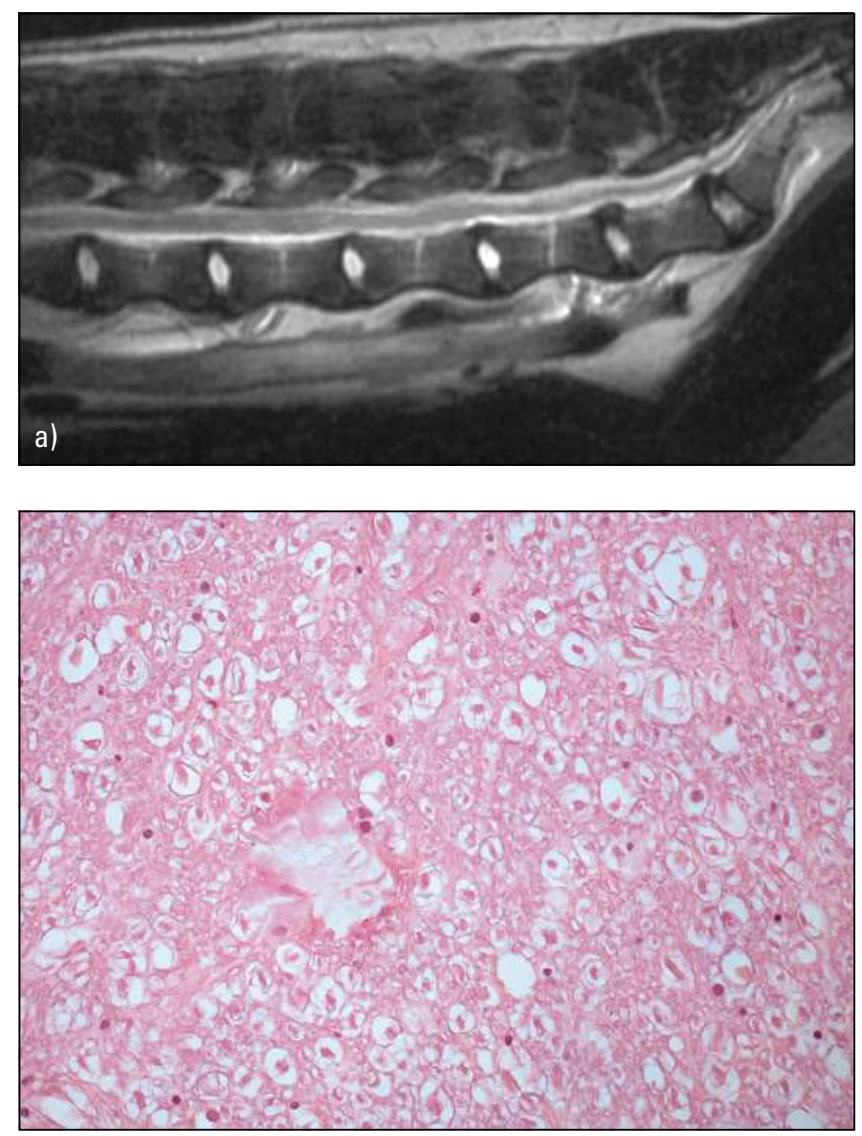

b)

Nr. 2, 4, 14; Abb. 5, 6). Zwei dieser Patienten (Nr. 2, 14) wiesen in der T2-gewichteten TSE-Sequenz eine intramedulläre Hyperintensität auf, ein Hund (Nr. 4) ein normointenses Rückenmark (Abb. 4). In den drei verbliebenen Fällen (Hund Nr. 19, 21 und 22) wurden im Bereich von L1-4 (Hund Nr. 19), L4-6 (Hund Nr. 22) oder im Bereich des thorakolumbalen Übergangs (Hund Nr. 21) Befunde erhoben, die für das Vorliegen einer akuten ischämischen Myelomalazie sprechen. Ein fibrokartilaginärer Embolus konnte jedoch nicht nachgewiesen werden.

Bei allen vier Hunden mit explosiven Bandscheibenvorfällen zeigte sich intraoperativ ein deutliches Ödem mit einer Blaufärbung des Rückenmarks im Bereich der Hyperintensität in der T2-gewichteten TSE-Sequenz. In allen Fällen wurde eine Durotomie durchgeführt und das Rückenmark als gering- bis hochgradig malazisch befundet. Die beiden Hunde mit hochgradiger Malazie wurden auf Wunsch der Besitzer euthanasiert und histopathologisch untersucht. In beiden Fällen ließen sich eine hochgradige Myelomalazie und chondroides Diskusmaterial im Rückenmark nachweisen (Abb. 3), bei einem Tier zudem eine hochgradige Blutung und Knorpelgewebe in den Blutkoagula. Die Verdachtsdiagnose eines explosiven Bandscheibenvorfalls wurde somit bestätigt.
Abb. 4 a) Magnetresonanztomographische Darstellung der lumbalen Wirbelsäule in der sagittalen Projektionsebene in der T2-gewichteten TSE-Sequenz von Hund Nr. 4. Das Rückenmark ist normointens. b) Histopathologische Darstellung von fibrokartilaginärem Material in einem Gefäß in der weißen Substanz auf Höhe von L4 (HE-Färbung, $200 \times$, Originalvergrößerung). c) Im angrenzenden Gewebe der weißen Substanz einzelne geschwollene Achsenzylinder (Sphäroide, Pfeil) sowie Myelophagen in so genannten "digestion chambers" (Pfeilspitze) (HE-Färbung, $400 \times$, Originalvergrößerung).

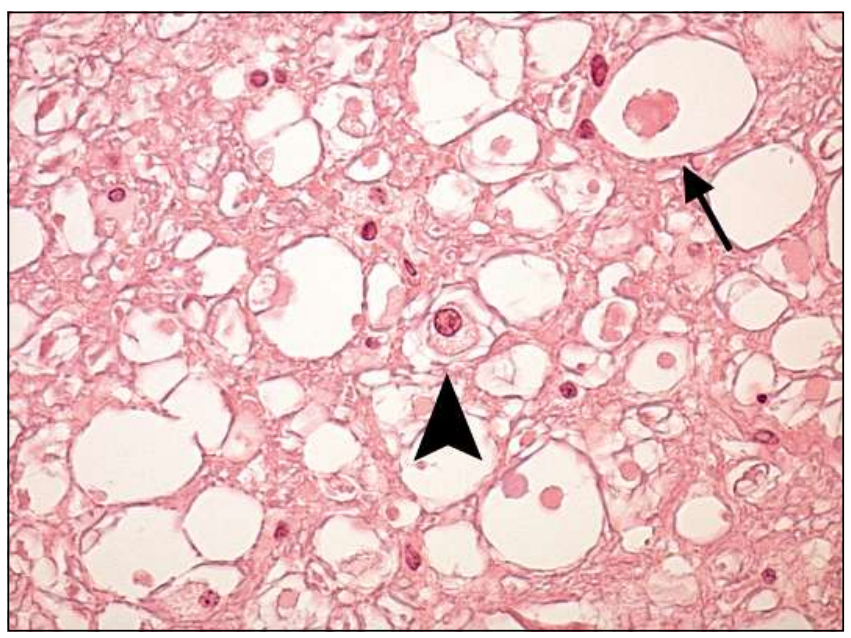

c)

\section{Diskussion}

Der fibrokartilaginäre Rückenmarksinfarkt stellt beim Hund eine relativ häufige Ursache für akute Lähmungen der Vorder- und Hintergliedmaßen dar. Außer beim Hund $(2,10,12,14)$ wurde der FCE auch bei Katze (1, 24, 25, 29), Pferd (30), Schwein (34) und Rind (9) beschrieben.

Einer Ischämie des Rückenmarks können neben einem fibrokartilaginären Embolus auch andere Ursachen zugrunde liegen, wie z. B. ein vaskulärer Verschluss durch Atherosklerose, Thromben, bakterielle, parasitäre oder neoplastische Emboli $(9,26)$. Die Pathophysiologie des fibrokartilaginären Rückenmarksinfarktes ist nicht geklärt und die verschiedenen Hypothesen werden kontrovers diskutiert (zusammengefasst z. B. bei 9). Nach Verschluss einer Arterie oder Vene durch einen fibrokartilaginären Embolus kommt es zur lokalen Ischämie. Als sekundäre pathophysiologische Reaktionen können die Neurone ihren aeroben Metabolismus nicht aufrechterhalten und verlieren ihre Membranpolarität. Kalzium und Natrium gelangen in die Zelle, während Kaliumionen aus der Zelle diffundieren. Wasser folgt dem osmotischen Gradienten in die Zelle und es resultiert ein intrazelluläres oder zytotoxisches Ödem (5). Durch den anaeroben Stoffwechsel akkumulieren Laktatmetaboliten, die den $\mathrm{pH}$-Wert der Zelle ernied- 

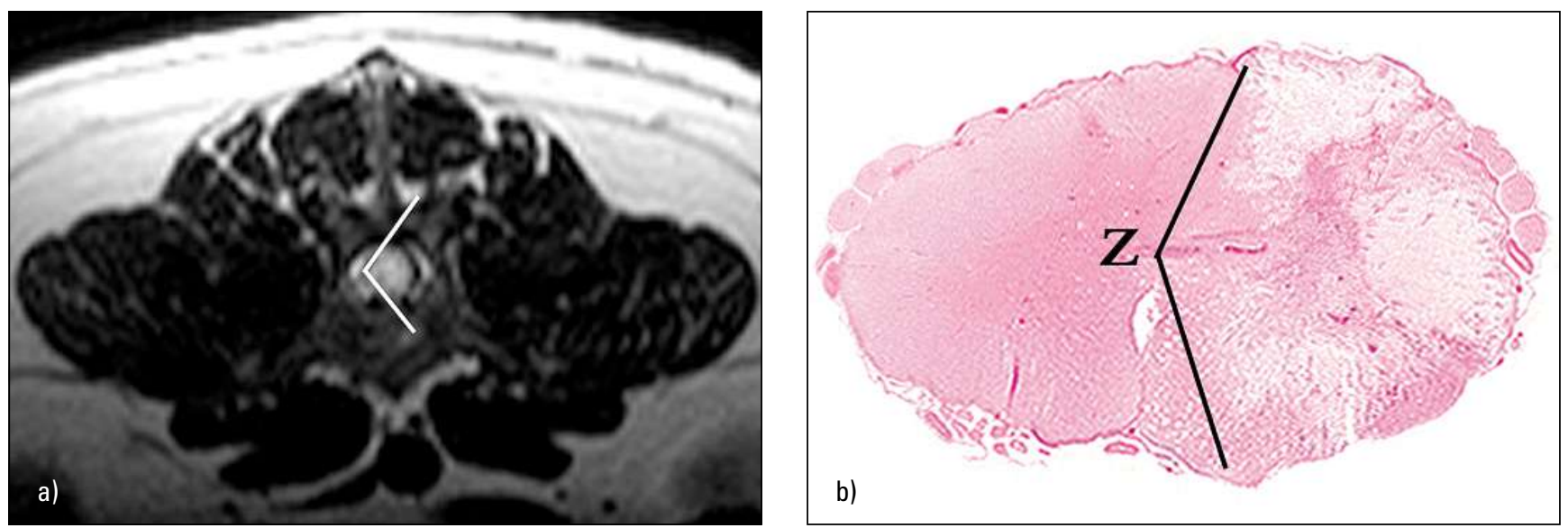

Abb. 5 a) Magnetresonanztomographische Darstellung der lumbalen Wirbelsäule in der transversalen Projektionsebene in der T2-gewichteten TSE-Sequenz von Hund Nr. 14. Die intramedulläre Hyperintensität, die die graue und weiße Substanz des Rückenmarks der rechten Seite betrifft, ist deutlich erkennbar. b) Mikroskopische Übersichtsdarstellung des Transversalschnitts durch das fixierte Rückenmark auf Höhe von L5/6 (HE-Färbung, Originalvergrößerung $5 \times$ ). Einseitige, hochgradige multifokale Malazie der weißen Substanz der dorsalen, ventralen und lateralen Funiculi infolge einer ischämischen Nekrose, verursacht durch einen fibrokartilaginären Embolus. $Z=$ Zentralkanal.
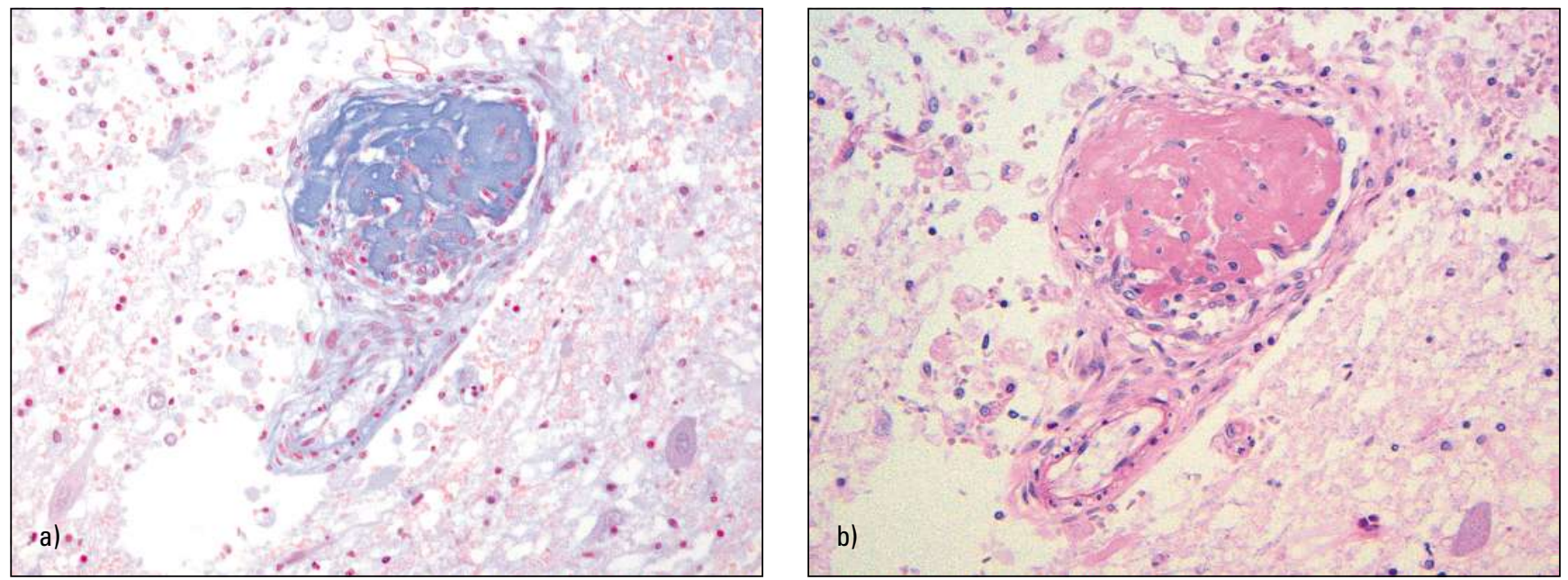

Abb. 6 Querschnitt durch das Rückenmark eines Hundes (Nr. 14) mit durch einen fibrokartilaginären Embolus ausgelösten Rückenmarksinfarkt in der Alzianblau-Färbung (a) und der PAS-Färbung (b) (Originalvergrößerung $100 \times$ ). Fibrokartilaginärer Embolus in einem Gefäß der weißen Substanz mit Malazie im angrenzenden Gewebe. Das fibrokartilaginäre Material stellt sich in der Alzianblau-Färbung blau und in der PAS-Färbung leuchtend rot dar.

rigen. Gleichzeitig werden Zytokine sowie exzitatorische Aminosäuren (Glutamat und Aspartat) freigesetzt und freie Radikale (z. B. Superoxid, Hydrogenperoxid und Stickstoffmonoxid) gebildet, die die Zellen angreifen und zur parenchymalen Malazie führen (15).

Der fibrokartilaginäre Rückenmarksinfarkt kommt häufig bei großen Hunderassen vor. Ein höherer Druck im Nucleus pulposus und größere Blutgefäße werden hierfür verantwortlich gemacht (12). Zwergschnauzer und junge Irische Wolfshunde scheinen eine Prädisposition für diese Erkrankung zu haben $(20,21)$. In der präsentierten Studie gehörten ca. 90\% der Hunde zu den großen Hunderassen (Körpergewicht über $20 \mathrm{~kg}$ ).

Der fibrokartilaginäre Rückenmarksinfarkt kann bereits ab einem Alter von acht Wochen auftreten, wird jedoch am häufigsten beim erwachsenen Hund in einem Alter zwischen drei und sechs Jahren beschrieben $(9,10,21,26)$. Der Altersdurchschitt der Hunde der vorliegenden Studie war mit 6,5 Jahren somit relativ hoch.

Die neurologischen Symptome hängen von der anatomischen Lokalisation des Infarktes und der Schwere der Ischämie 
Magnetresonanztomographische Befunde bei Hunden mit Verdacht auf fibrokartilaginären Rückenmarksinfarkt V. M. Stein, F. Wagner, C. Bull, A. Gerdwilker, F. Seehusen, W. Baumgärtner, A. Tipold

ab. Rückenmarksinfarkte können fokal im Bereich der gesamten Länge des Rückenmarks auftreten. Aufgrund der Gefäßverteilung und -dichte sind häufig die Intumeszenzen betroffen $(9,13)$, was Störungen des unteren motorischen Neuronsystems (abwesende oder herabgesetzte Reflexe) hervorruft. In dieser Studie waren das obere motorische Neuronsystem (T3-L3) und das untere motorische Neuronsystem (L4-S3) in ausgeglichener Anzahl die am häufigsten vertretenen Läsionen. Summers et al. (32) beschreiben, dass ca. $60 \%$ der Fälle mit Lähmungen der Hintergliedmaßen, also einer Läsion kaudal von T3, einhergehen. In dem beschriebenen Patientengut waren es sogar ca. 80\% der Hunde, die eine Parese oder Plegie der Hintergliedmaßen zeigten. Häufig wird eine Asymmetrie der neurologischen Defizite gesehen (9), bei der die nicht betroffene Seite sogar unauffällig sein kann (26). Dies liegt darin begründet, dass die A. spinalis ventralis die Aa. sulcocommissurales entlässt, von der die graue Substanz und Areale der weißen Substanz versorgt werden. Dieser Gefäßabgang erfolgt segmental zu beiden Seiten oder alternativ zu einer oder der anderen Seite $(13,27)$. Erfolgt eine Embolisation im Bereich des einseitigen Gefäßabgangs, bedingt dies eine Ischämie in einer Hälfte des Rückenmarks (Abb. 5a und b). So ist es möglich, dass einzelne Quadranten des Rückenmarks betroffen sind, während andere in derselben Region völlig unverändert sind $(11,26)$. Eine Studie fand eine klinische Asymmetrie in ca. 55-65\% der Fälle (17), was sich mit den Befunden unserer Studie mit asymmetrischen Defiziten bei ca. 70\% der Hunde deckt. Die klinische Asymmetrie korrelierte sehr gut mit den Befunden der magnetresonanztomographischen Transversalschnitte, die seitenbetonte Hyperintensitäten darstellten. Oftmals wird berichtet, dass die initiale Asymmetrie zu symmetrischen Ausfällen fortgeschritten ist, was durch das sich entwickelnde zytotoxische Ödem im Rückenmarksparenchym erklärt werden kann (9).

Der fibrokartilaginäre Rückenmarksinfarkt war lange eine Ausschlussdiagnose nach unauffälliger Myelographie oder der Darstellung geringgradig ausgedünnter Kontrastmittelsäulen im Bereich der Läsion (9). Beim Menschen wird die Magnetresonanztomographie bereits seit 1989 in der Diagnostik von Rückenmarksinfarkten eingesetzt (7). Seit dem Einzug der MRT in die Veterinärmedizin stellt sich die Frage, ob mit diesem wenig invasiven bildgebenden Verfahren der fibrokartilaginäre Rückenmarksinfarkt darstellbar und somit nachweisbar wird. Obwohl viele veterinärmedizinische Autoren den Einsatz der MRT bei einem entsprechenden Verdacht empfehlen $(9,26,31)$, gibt es kaum Angaben über die Befunde. Erst in jüngerer Zeit sind Publikationen über die MRT-Diagnostik bei Katzen und Hunden mit fibrokartilaginären Rückenmarksinfarkten erschienen. Als Befunde werden ein Ödem und eine Schwellung des Rückenmarks im Bereich der Läsion beschrieben (31), die sich in der T2-gewichteten TSE-Sequenz als intramedulläre Hyperintensität darstellen (2, 19, 24, 25, 35). Die Darstellung nach intravenöser Kontrastmittelgabe hängt vom Alter des Infarktes ab. In der frühen Phase nach Beginn der neurologischen Symptome wurde entweder keine oder eine milde Kontrastmittelanreicherung beobachtet $(2,19)$. Diese konstante Darstellung des fibrokartilaginären Rückenmarksin-
Tab. 3 Mögliche Einflussfaktoren auf die Darstellung von fibrokartilaginären Rückenmarksinfarkten in der T2-gewichteten TSESequenz. OMN = oberes motorisches Neuronsystem, UMN = unteres motorisches Neuronsystem.

\begin{tabular}{|c|c|c|c|}
\hline \multirow[t]{2}{*}{ Charakteristikum } & \multirow[t]{2}{*}{ Anzahl } & \multicolumn{2}{|l|}{ T2 } \\
\hline & & hyperintens & normointens \\
\hline \multicolumn{4}{|l|}{ Gefäßtopographie } \\
\hline $\begin{array}{l}\text { Läsion OMN } \\
\text { (C1-C5; T3-L3) }\end{array}$ & 10 & 7 & 3 \\
\hline $\begin{array}{l}\text { Läsion UMN } \\
\text { (C6-T2; L4-S3) }\end{array}$ & 12 & 7 & 5 \\
\hline $\begin{array}{l}\text { einmalige Glukokortiko- } \\
\text { steroidgabe }\end{array}$ & 15 & 9 & 6 \\
\hline $\begin{array}{l}\text { Schwere der motori- } \\
\text { schen Ausfälle }\end{array}$ & 22 & $\begin{array}{l}14 \\
7 \text { Plegie } \\
7 \text { Parese }\end{array}$ & $\begin{array}{l}8 \\
1 \text { Plegie } \\
7 \text { Parese }\end{array}$ \\
\hline Verlauf & 13 & $\begin{array}{l}8 \\
\text { Besserung } \\
\text { nach } 12,5 \mathrm{~d}\end{array}$ & $\begin{array}{l}5 \\
\text { Besserung } \\
\text { nach } 4,2 \mathrm{~d}\end{array}$ \\
\hline \multicolumn{4}{|l|}{$\begin{array}{l}\text { Zeit zwischen Beginn } \\
\text { neurologischer Ausfälle } \\
\text { und MRT }\end{array}$} \\
\hline$<24 \mathrm{~h}$ & 11 & 8 & 3 \\
\hline $24-48 \mathrm{~h}$ & 5 & 3 & 2 \\
\hline$>48 \mathrm{~h}$ & 6 & 3 & 3 \\
\hline
\end{tabular}

farktes als intramedulläre Hyperintensität in der T2-gewichteten TSE-Sequenz deckt sich nicht mit den Resultaten der hier präsentierten Studie.

In der vorliegenden Untersuchung wurde bei allen 26 Hunden eine extradurale Kompression ausgeschlossen. Bei 18 Tieren zeigte sich in der T2-gewichteten TSE-Sequenz eine hyperintense Läsion (davon 14 Hunde mit Verdacht auf fibrokartilaginären Rückenmarksinfarkt und vier Hunde mit explosiven Bandscheibenvorfällen), während sich bei acht Hunden mit Verdacht auf einen Rückenmarksinfarkt das Rückenmark - entgegen den Beschreibungen in der Literatur - normointens darstellte. Obwohl die klinische Präsentation sehr typisch für das Vorliegen eines Rückenmarksinfarktes war, kann die Hyperintensität in der T2-gewichteten TSE-Sequenz fehlen. Diese Feststellung wird dadurch untermauert, dass bei einem Hund (Nr. 4) mit normointensem Rückenmark das fibrokartilaginäre Material in den Arterien als Ursache der Lähmungserscheinungen histopathologisch nachgewiesen werden konnte. Welche potenziellen Einflussfaktoren sowie Konsequenzen für das Vorhandensein oder Fehlen der intramedullären Hyperintensität infrage kommen, wurde evaluiert und soll im Weiteren diskutiert werden.

So könnten die neuroanatomische Lokalisation unter Berücksichtigung der Gefäßtopographie und die Größe des embolisierten Gefäßes eine wichtige Rolle für das Auftreten einer Hyperintensität in der T2-gewichteten TSE-Sequenz haben. Das Rücken-

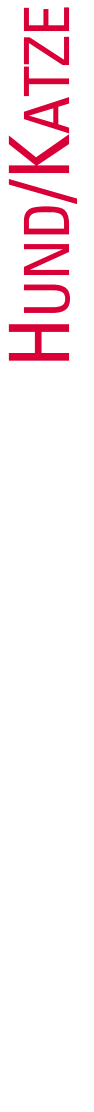


Magnetresonanztomographische Befunde bei Hunden mit Verdacht auf fibrokartilaginären Rückenmarksinfarkt V. M. Stein, F. Wagner, C. Bull, A. Gerdwilker, F. Seehusen, W. Baumgärtner, A. Tipold

mark ist durch extensive Gefäßanastomosen in den leptomeningealen Gefäßen gut vor ischämischen Insulten geschützt. Die intrinsischen, also parenchymalen Gefäße stellen jedoch eine arterielle Endstrombahn dar (11). Die unterschiedliche Gefäßanatomie in den verschiedenen Bereichen des Rückenmarks mag zudem einen wichtigen Einfluss auf die Vulnerabilität haben. Im thorakalen Bereich wurden die geringste Anzahl an Radikulararterien und die im Durchmesser kleinsten Gefäße gefunden (8). Zudem ist hier die Anzahl der Aa. sulcocommissurales pro Zentimeter signifikant geringer als im zervikalen oder lumbalen Rückenmarksabschnitt (9). Es kann daher zwar angenommen werden, dass eine Embolisation in einem Bereich mit geringerer Gefäßdichte seltener auftritt als in Bereichen mit größerer Gefäßdichte. Andererseits ist jedoch auch anzunehmen, dass sich in einem Bereich mit geringerer Gefäßdichte ein dramatischerer Effekt für das Rückenmark ergibt, wenn eines dieser wenigen Gefäße verschlossen wird (9). Im Einklang mit dieser Überlegung steht, dass in der vorliegenden Studie die deutliche Mehrzahl der Tiere mit einer Läsion bei T3-L3 eine intramedulläre Hyperintensität zeigte.

Das Vorhandensein oder Fehlen einer Hyperintensität in der T2-gewichteten TSE-Sequenz schien nicht in Zusammenhang mit einer zuvor erfolgten Glukokortikosteroidgabe zu stehen, da sowohl die vorbehandelten als auch die nicht vorbehandelten Tiere hyperintense Läsionen oder ein normointenses Rückenmark aufwiesen. Abramson et al. (2) beschreiben, dass eine einmalige Steroidgabe vor der Überweisung nicht ausreicht, um eine entzündliche Erkrankung des Rückenmarks in der Bildgebung völlig zu maskieren. Es kann also davon ausgegangen werden, dass die einmalige Glukokortikosteroidapplikation keinen wesentlichen Einfluss auf die MRT-Darstellung eines intramedullären Ödems hat.

Die Zeitspanne vom akuten Auftreten neurologischer Ausfallerscheinungen bis zur MRT-Diagnostik könnte einen Einfluss auf die Darstellbarkeit der Läsion haben. Abramson et al. (2) stellten fest, dass die Dauer der Symptomatik in der T1-SE-Sequenz die Darstellung der Läsion und die Anreicherung mit Kontrastmittel beeinflusst, während sich in der T2-TSE-Sequenz konstant eine Hyperintensität zeigte. Nach unseren Ergebnissen besteht innerhalb der ersten 48 Stunden kein offensichtlicher Einfluss. Im Vergleich zur Untersuchung von Abramson et al. (2) wurden die Hunde der vorliegenden Studie generell früher einer MRT-Diagnostik unterzogen. Auch Erkenntnisse aus der Humanmedizin lassen eine zeitliche Abhängigkeit der Darstellung eines Rückenmarksinfarktes vermuten (16). So beschreiben Weidauer et al. (36) das Fehlen einer intramedullären Hyperintensität bei Patienten, die besonders zeitnah zur Initiation der Lähmungen magnetresonanztomographisch untersucht wurden. In MRT-Folgeuntersuchungen nach fünf und acht Tagen konnte jedoch auch bei diesen Patienten eine intramedulläre Hyperintensität dargestellt werden. Ein Einfluss der Zeitspanne zwischen dem Beginn neurologischer Ausfallerscheinungen und den MRT-Befunden ist schwierig zu bestimmen, da hierzu eine Kontrolluntersuchung zu einem späteren Zeitpunkt erforderlich wäre. Bei veterinärmedizinischen Patienten mit normointensem Rückenmark ist jedoch selten eine Indikation zur MRT-Kontrolluntersuchung gegeben, die eine erneute Allgemeinanästhesie des Patienten rechtfertigen würde.

In Bezug auf die Schwere der neurologischen Lähmungserscheinungen zeigte sich, dass Hunde mit einer hyperintensen Läsion in der T2-TSE-Sequenz unterschiedlich starke Lähmungserscheinungen aufweisen können. Hunde mit normointensem Rückenmark scheinen tendenziell weniger schwerwiegende Lähmungserscheinungen zu zeigen. Im Zusammenhang damit ist die neurologische Besserung der Patienten zu sehen, die bei Hunden mit normointensem Rückenmark tendenziell schneller fortschritt als bei Tieren mit einer hyperintensen intramedullären Läsion. Es liegt die Vermutung nahe, dass bei Hunden mit normointensem Rückenmark eine geringere oder weniger ausgedehnte Schädigung des Rückenmarks vorliegt als bei Hunden mit intramedullärer Hyperintensität und sie daher weniger deutlich zu sehen ist.

Der Status der Nuclei pulposi in der Nachbarschaft der ischämischen Myelopathie ist bisher nicht genau untersucht. In manchen Fällen erschienen sie unverändert, während in anderen Fällen röntgenologisch oder durch die pathologische Untersuchung Hinweise auf eine Degeneration bestanden $(11,32)$. Besonders interessant ist die Beschreibung von Grünenfelder et al. (19). Bei initialer MRT-Diagnostik konnten diese Autoren bei einem Hund mit fibrokartilaginärem Rückenmarksinfarkt keinen Hinweis auf eine Diskusdegeneration feststellen, während sich bei einer MRT-Kontrolluntersuchung nach 14 Wochen dieser Diskus in direkter Nachbarschaft der intramedullären Hyperintensität degeneriert zeigte. Normalerweise stellen sich die Nuclei pulposi in der T2-TSE-Sequenz mit hoher Signalintensität dar (3). Ein partieller Verlust dieser Signalintensität indiziert wahrscheinlich eine Degeneration im Sinne einer Dehydration oder Herniation des Diskus. In unserer Studie wiesen sieben Hunde eine Degeneration der Disci intervertebrales in direkter Nachbarschaft der Hyperintensität in der T2-gewichteten TSE-Sequenz auf. Lediglich bei vier Hunden mit degenerierten Bandscheiben befanden sich diese mehr als zwei Zwischenwirbelspalten entfernt von der Läsion. Es liegt die Vermutung nahe, dass ein Zusammenhang zwischen degenerierten Nuclei pulposi und Lokalisation des Rückenmarksinfarktes besteht.

Die definitive Diagnose eines fibrokartilaginären Infarktes liefert die histopathologische Untersuchung. Als charakteristischer Befund findet sich eine ischämische Myelopathie im Bereich der Läsion. Ein diagnostischer Befund ist die Darstellung fibrokartilaginären Materials, das die Arterien oder Venen des Rückenmarks partiell oder komplett verschließt und histologisch sowie histochemisch mit dem Nucleus pulposus identisch ist (6, 12). Die Emboli färben sich positiv für Mukopolysaccharide an, d. h. blau in der Alzianblau-Färbung und leuchtend rot/magenta in der PAS-(Periodic-acid-Schiff-)Färbung (9, 32; Abb. 6). Als Folge der lokalen Ischämie entwickeln sich Nekrosen oder Malazien der grauen und weißen Substanz. Diese zeigen sich bereits bei geringer Vergrößerung als deutliche Vakuolisierung des Parenchyms (Abb. 4b). Das Bild wird zudem durch eine vaskuläre Nekrose, axonale Degeneration sowie unterschiedlich starke Abräumreaktionen durch lipidbeladene Makrophagen und neutrophile Infiltrate geprägt $(26,32)$. 
Magnetresonanztomographische Befunde bei Hunden mit Verdacht auf fibrokartilaginären Rückenmarksinfarkt

V. M. Stein, F. Wagner, C. Bull, A. Gerdwilker, F. Seehusen, W. Baumgärtner, A. Tipold

Die Differenzialdiagnosen zum fibrokartilaginären Rückenmarksinfarkt sind nach der Diagnostik mittels MRT deutlich reduziert. Sie wurden in dieser Studie erstmalig ausgewertet. Dabei stellte sich heraus, dass lediglich explosive Bandscheibenvorfälle, die einen ebenso akuten bis perakuten Beginn der Symptomatik aufweisen wie die Rückenmarksinfarkte, oftmals asymmetrische und nicht progressive Lähmungen hervorrufen und häufig ohne Schmerzhaftigkeit verlaufen $(18,10)$, nicht sicher von fibrokartilaginären Rückenmarksinfarkten unterschieden werden konnten. Eine Differenzierung der vier Fälle von explosiven Bandscheibenvorfällen erfolgte aufgrund der intraoperativen oder histopathologischen Befunde. Dem akuten explosiven und nicht kompressiven Bandscheibenvorfall misst auch Bagley (4) eine besondere Bedeutung als Differenzialdiagnose des fibrokartilaginären Rückenmarksinfarktes bei und sieht ihn als häufige Ursache einer Fehldiagnose. Als weitere Differenzialdiagnose kommt eine Rückenmarkskontusion in Betracht, die wie der fibrokartilaginäre Rückenmarksinfarkt eine fokale Hyperintensität in der T2-gewichteten MRT-Sequenz hervorruft und keine Kontrastmittelanreicherung zeigt $(4,9)$. Die Differenzierung dieser pathologischen Veränderungen des Rückenmarks mittels MRT ist schwierig bis unmöglich, da sie mit einem intramedullären Ödem einhergehen können $(4,28)$. Die Diagnose eines fibrokartilaginären Rückenmarksinfarktes bleibt somit auch mit dem Einsatz der Magnetresonanztomographie lediglich eine Verdachtsdiagnose.

\section{Fazit für die Praxis}

Die präsentierte Studie mit 22 Hunden mit der Verdachtsdiagnose eines fibrokartilaginären Rückenmarksinfarktes liefert wichtige Erkenntnisse über die magnetresonanztomographische Darstellung der Läsion. Insbesondere wird deutlich, dass der fehlende Nachweis einer intramedullären hyperintensen Läsion in der T2-gewichteten TSE-Sequenz einen fibrokartilaginären Infarkt nicht ausschließt. Umgekehrt ist jedoch auch der Nachweis einer intramedullären hyperintensen Läsion in der T2-gewichteten TSE-Sequenz kein Beweis für das Vorliegen eines fibrokartilaginären Rückenmarksinfarktes, da sich der explosive Bandscheibenvorfall ähnlich darstellt. Somit ist es weiterhin wichtig, dass Vorbericht und Signalement des Hundes sowie die neurologischen Befunde im Zusammenspiel ein Bild ergeben, die das Vorliegen eines Rückenmarksinfarktes sehr wahrscheinlich machen. Die MRT-Darstellung des fibrokartilaginären Rückenmarksinfarktes kann in der T2-gewichteten TSE-Sequenz hyperintens sein oder das Rückenmark zeigt sich im Bereich der neuroanatomischen Lokalisation normointens. Das Vorhandensein oder Fehlen einer intramedullären Hyperintensität könnte durch die Zeitspanne vom Auftreten der neurologischen Lähmungserscheinungen bis zur Durchführung der MRT-Diagnostik sowie von der Größe und Lokalisation des embolisierten Gefäßes entscheidend beeinflusst werden.

\section{Danksagung}

Wir möchten uns bei den Kollegen und Kolleginnen für ihre freundlichen Überweisungen der Patienten herzlich bedanken sowie bei Dr. Irene Böttcher und Dr. Thilo von Klopmann für die Hilfe bei der stationären Betreuung der Tiere. Diese Studie wurde durch die Frauchiger Stiftung, Bern finanziell unterstützt.

\section{Literatur}

1. Abramson CJ, Platt SR, Stedman NL. Tetraparesis in a cat with fibrocartilaginous emboli. J Am Anim Hosp Assoc 2002; 38: 153-156.

2. Abramson CJ, Garosi L, Platt SR, Dennis R, Fraser McConnell J. Magnetic resonance imaging appearance of suspected ischemic myelopathy in dogs. Vet Radiol Ultrasound 2005; 46: 225-229.

3. Adams WH, Daniel GB, Pardo AD, Selcer RR. Magnetic resonance imaging of the caudal lumbar and lumbosacral spine in 13 dogs (1990-1993). Vet Radiol Ultrasound 1995; 36: 3-13.

4. Bagley RS. Spinal cord enigmas: Fibrocartilaginous emboli, arachnoid cyst, and others. 21st Annual ACVIM Forum Proceedings, Charlotte, NC, 2003; $10-11$.

5. Bagley RS. Pathophysiology in nervous system disease. In: Fundamentals of Veterinary Clinical Neurology. Bagley RS, ed. Oxford: Blackwell Publishing 2005; 45.

6. Bichsel P, Vandevelde M, Lang J. L'infarctus de la moelle épinière à la suite d'embolies fibrocartilagineuses chez le chien et le chat. Schweiz Arch Tierheilk 1984; 126: 387-397.

7. Brown E, Virapongse C, Gregorios JB. MR imaging of cervical cord infarction. J Comp Assist Tomogr 1989; 13: 920-922.

8. Caulkins SE, Purinton PT, Oliver JE. Arterial supply to the spinal cord of dogs and cats. Am J Vet Res 1989; 50: 425-430.

9. Cauzinille L. Fibrocartilaginous embolism in dogs. Vet Clin North Am: Small Anim Pract 2000; 30: 155-167.

10. Cauzinille L, Kornegay JN. Fibrocartilaginous embolism of the spinal cord in dogs: review of 36 histologically confirmed cases and retrospective study of 26 suspected cases. J Vet Intern Med 1996; 10: 241-245.

11. Cook JR. Fibrocartilaginous embolism. Vet Clin North Am: Small Anim Pract 1988; 18: 581-592.

12. deLahunta A, Alexander JW. Ischemic myelopathy secondary to presumed fibrocartilaginous embolism in nine dogs. J Am Anim Hosp Assoc 1976; 12: $37-48$.

13. deLahunta A. Veterinary Neuroanatomy and Clinical Neurology. Philadelphia: Saunders 1983.

14. Dyce J, Houlton JEF. Fibrocartilaginous embolism in the dog. J Small Anim Pract 1993; 34: 332-336.

15. Evans RW, Wilberger JE. Traumatic disorders. In: Textbook of Clinical Neurology. Goetz CG, Pappert EJ, eds. Philadelphia: Saunders 1999; $1035-1058$

16. Fortuna A, Ferrante L, Acqui M, Trillo G. Spinal cord ischemia diagnosed by MRI. J Neuroradiol 1995; 22: 115-122.

17. Gandini G, Cizinauskas S, Lang J, Fatzer R, Jaggy A. Fibrocartilaginous embolism in 75 dogs: clinical findings and factors influencing the recovery rate. J Small Anim Pract 2003; 44: 76-80.

18. Griffiths IR. A syndrome produced by dorso-lateral „explosions“ of the cervical intervertebral discs. Vet Rec 1970; 87: 737-741.

19. Grünenfelder FI, Weishaupt D, Green R, Steffen F. Magnetic resonance imaging findings in spinal cord infarction in three small breed dogs. Vet Radiol Ultrasound 2005; 46: 91-96.

20. Hawthorne JC, Wallace LJ, Fenner WR, Waters DJ. Fibrocartilaginous embolic myelopathy in Miniature Schnauzers. J Am Anim Hosp Assoc 2001; 37 : 374-383.

21. Junker K, Van Den Ingh TSGAM, Bossard MM, Van Nes JJ. Fibrocartilaginous embolism of the spinal cord (FCE) in juvenile Irish Wolfhounds. Vet Quart 2000; 22: 154-156.

22. Kippenes H, Gavin PR, Bagley RS, Silver GM, Tucker RL, Sande RD. Magnetic resonance imaging features of tumors of the spine and spinal cord in dogs. Vet Radiol Ultrasound 1999; 40: 627-633. 
Magnetresonanztomographische Befunde bei Hunden mit Verdacht auf fibrokartilaginären Rückenmarksinfarkt

V. M. Stein, F. Wagner, C. Bull, A. Gerdwilker, F. Seehusen, W. Baumgärtner, A. Tipold

23. Kornegay JN. Ischemic myelopathy due to fibrocartilaginous embolism. Comp Cont Educ Pract Vet 1980; 2: 402-406.

24. MacKay AD, Rusbridge C, Sparkes AH, Platt SR. MRI characteristics of suspected acute spinal cord infarction in two cats, and a review of the literature. J Fel Med Surg 2004; 7: 101-107.

25. Mikszewski JS, Van Winkle TJ, Troxel MT. Fibrocartilaginous embolic myelopathy in five cats. J Am Anim Hosp Assoc 2006; 42: 226-233.

26. Neer TM. Fibrocartilaginous emboli. Vet Clin North Am: Small Anim Pract 1992; 22: 1017-1025.

27. Nickel R, Schummer A, Seiferle E, Böhme, G. Lehrbuch der Anatomie der Haustiere, Band 4. Nervensystem, Sinnesorgane, Endokrine Drüsen. Berlin, Hamburg: Parey 2004.

28. Sanders SG, Bagley RS, Gavin PR. Intramedullary spinal cord damage associated with intervertebral disk material in a dog. J Am Vet Med Assoc 2002;1574-1575, 1594-1596.

29. Scott HW, O'Leary MT. Fibrocartilaginous embolism in a cat. J Small Anim Pract 1996; 37: 228-231.

30. Sebastian MM, Giles RC. Fibrocartilaginous embolic myelopathy in a horse. J Vet Med A 2004; 51: 341-343.

31. Sharp NJH, Wheeler SJ. Fibrocartilaginous embolism. In: Small Animal Spinal Disorders. London, New York: Elsevier Mosby 2005; 332.

32. Summers B, Cummings JF, deLahunta A. Veterinary Neuropathology. St. Louis: Mosby 1995.
33. Tartarelli CL, Baroni M, Borghi M. Thoracolumbar disc extrusion associated with extensive epidural haemorrhage: a retrospective study of $23 \mathrm{dogs}$. J Small Anim Pract 2005; 46: 485-490.

34. Tessaro SV, Doige CE, Rhodes CS. Posterior paralysis due to fibrocartilaginous embolism in two weaner pigs. Can J Comp Med 1983; 47: 124-126.

35. Ueno H, Shimizu J, Uzuka Y, Kobayashi Y, Hirokawa H, Ueno E, Suzuki A, Yamada K. Fibrocartilaginous embolism in a chondrodystrophoid breed dog. Aust Vet J 2005; 83: 142-144.

36. Weidauer S, Nichtweiss M, Lanfermann H, Zanelle FE. Spinal cord infarction: MR imaging and clinical features in 16 cases. Neuroradiology 2002; 44 : 851-857.

Dr. Veronika M. Stein, Ph. D.

Klinik für Kleintiere

Stiftung Tierärztliche Hochschule Hannover

Bischofsholer Damm 15

30173 Hannover

E-Mail: Veronika.Stein@tiho-hannover.de 